\title{
SUMS OF PRODUCTS OF POSITIVE OPERATORS AND SPECTRA OF LÜDERS OPERATORS
}

\author{
BOJAN MAGAJNA \\ (Communicated by Richard Rochberg)
}

\begin{abstract}
Each bounded operator $T$ on an infinite dimensional Hilbert space $\mathcal{H}$ is a sum of three operators that are similar to positive operators; two such operators are sufficient if $T$ is not a compact perturbation of a scalar. The spectra of Lüders operators (elementary operators on $\mathrm{B}(\mathcal{H})$ with positive coefficients) of lengths at least three are not necessarily contained in $\mathrm{B}(\mathcal{H})^{+}$. On the other hand, the spectra of such operators of lengths (at most) two are contained in $\mathrm{B}(\mathcal{H})^{+}$if the coefficients on one side commute.
\end{abstract}

\section{INTRODUCTION}

Completely positive maps on $\mathrm{B}(\mathcal{H})$ (the algebra of all bounded operators on a Hilbert space $\mathcal{H}$ ) of the form

$$
\Psi(X)=\sum_{j=1}^{n} A_{j}^{*} X A_{j}
$$

have received renewed interest recently especially in connection with quantum information theory (see [8], 9], [13, 18] and the references there). If all the coefficients $A_{j}$ in (1.1) are positive operators such a map is called a Lüders operation. If $n$ is finite, then these are special cases of elementary operators, that is, maps of the form $X \mapsto \sum_{j=1}^{n} A_{j} X B_{j}$, whose spectra have been intensively studied in the past (see [5] and the references there), but only in the cases when both families of coefficients $\left(A_{j}\right)$ and $\left(B_{j}\right)$ are commutative. If $\mathcal{H}$ is finite dimensional, then $\mathrm{B}(\mathcal{H})$ is a Hilbert space for the inner product induced by the trace and it is easily verified that an elementary operator with positive coefficients $A_{j}$ and $B_{j}$ is a positive operator on this Hilbert space, so its spectrum is contained in $\mathbb{R}^{+}:=[0, \infty)$.

At the end of the paper [1] it was asked if the spectrum of a Lüders operator $X \mapsto \sum_{j=1}^{n} A_{j} X A_{j}$ with positive coefficients on $\mathrm{B}(\mathcal{H})$ is necessarily contained in $\mathbb{R}^{+}$if $\mathcal{H}$ is infinite dimensional. We will show that, contrary to what one might expect, the answer to this question is negative. This will be a consequence of the fact that the operator $T=-1$ can be expressed as

$$
T=\sum_{j=1}^{n} A_{j} B_{j} \text { with positive } A_{j}, B_{j} \in \mathrm{B}(\mathcal{H}) .
$$

Received by the editors August 19, 2011.

2010 Mathematics Subject Classification. Primary 47A05, 47B47; Secondary 47N50, 81P45.

Key words and phrases. Positive operators, commutators, quantum operations. 
At first the author did not know how to prove that every operator $T \in \mathrm{B}(\mathcal{H})$ is of the form (1.2), but then Professor Heydar Radjavi told him that by [16] and [12] $T$ is a sum of finitely many idempotents and, since every idempotent is similar to a projection, $T$ is a sum of products of positive operators. To see this, note that an operator $Q$ which is similar to a positive operator, say $Q=S P S^{-1}$, is a product of two positive operators: $Q=\left(S S^{*}\right)\left(\left(S^{-1}\right)^{*} P S^{-1}\right)$. By Pearcy and Topping 12 . five idempotents are sufficient to express any $T$ in this way, and according to 19 , Proposition 5.9] this is the minimal number since scalars are in general not sums of less than five idempotents. However, since idempotents are very special elements, we cannot expect that 5 is the minimal $n$ in (1.2).

One of the goals of this paper is to find the minimal $n$ above. The result will imply that even the spectrum of a Lüders operator of small length is not necessarily contained in $\mathbb{R}^{+}$. More precisely, in the next section we will show that every $T \in \mathrm{B}(\mathcal{H})$ is a sum of three operators $T_{j}$ each of which is similar to a positive operator. Moreover, if $T$ is not a compact perturbation of a scalar, two operators $T_{j}$ are sufficient. This result is optimal since compact perturbations of nonzero scalars cannot be expressed in the form (1.2) with $n \leq 2$. We will also show that the trace class operators with trace not in $\mathbb{R}^{+}$cannot be expressed as $T_{1}+T_{2}$ with both $T_{1}$ and $T_{2}$ similar to positive operators in $\mathrm{B}(\mathcal{H})$. As a preliminary step in the proof of the main result we will first show that $T$ is a sum of four operators $T_{j}$ similar to positive ones, with some additional properties needed.

In the last section we will first apply this result to answer the above-mentioned question from [11]. Then we will prove that the spectra of operators of the form $X \mapsto \sum_{j=1}^{2} A_{j} X B_{j}$ with positive $A_{j}$ and $B_{j}$ are contained in $\mathbb{R}^{+}$if $A_{1} A_{2}=A_{2} A_{1}$ (or if $B_{1} B_{2}=B_{2} B_{1}$ ).

Throughout the paper $\mathcal{H}$ denotes an infinite dimensional separable Hilbert space and $\mathrm{B}(\mathcal{H})$ the algebra of all bounded linear operators on $\mathcal{H}$. (The results hold also for nonseparable $\mathcal{H}$, but in their formulations the ideal of compact operators must be replaced by the unique proper maximal ideal of $\mathrm{B}(\mathcal{H})$.) An operator $T \in \mathrm{B}(\mathcal{H})$ is called positive if $\langle T \xi, \xi\rangle \geq 0$ for all $\xi \in \mathcal{H}$ (thus $T$ is not necessarily definite), and the set of all positive operators is denoted by $\mathrm{B}(\mathcal{H})^{+}$.

\section{SUMS OF OPERATORS SIMILAR TO POSITIVE OPERATORS}

We begin with a simple and well-known observation. Let $S \in \mathrm{B}(\mathcal{K} \oplus \mathcal{K})$ be a $2 \times 2$ operator matrix

$$
S=\left[\begin{array}{ll}
u & x \\
y & z
\end{array}\right]
$$

where $u$ is invertible. Then $S$ is invertible if and only if $z-y u^{-1} x$ is invertible and in this case

$$
S^{-1}=\left[\begin{array}{cc}
u^{-1}\left(1+x d y u^{-1}\right) & -u^{-1} x d \\
-d y u^{-1} & d
\end{array}\right], \quad \text { where } d=\left(z-y u^{-1} x\right)^{-1} .
$$

To prove this, multiply $S$ from the left by the invertible matrix

$$
\left[\begin{array}{cc}
u^{-1} & 0 \\
-y u^{-1} & 1
\end{array}\right]
$$

to obtain an upper-triangular matrix with 1 and $z-y u^{-1} x$ along the diagonal. 
The main assertion of the following lemma can be deduced from the proof of Theorem 1 in 12 , but later we will need some additional information from its proof in the form presented below.

Lemma 2.1. Every operator $T \in \mathrm{B}(\mathcal{H})$ is a sum of the form

$$
T=\sum_{j=1}^{4} S_{j} T_{j} S_{j}^{-1}
$$

where $S_{j} \in \mathrm{B}(\mathcal{H})$ and the operators $T_{j} \in \mathrm{B}(\mathcal{H})$ are positive with disjoint spectra $\sigma\left(T_{j}\right)$, and each $\sigma\left(T_{j}\right)$ consists of at most two points, $\sigma\left(T_{1}\right) \subset[0,1]$ and $\sigma\left(T_{j}\right) \subset$ $(1, \infty)$ for $j \neq 1$. Moreover, the range of $T_{1}$ is closed and has infinite dimension and codimension.

In particular, $T$ can be written as $T=\sum_{j=1}^{4} A_{j} B_{j}$, where $A_{j}, B_{j} \in \mathrm{B}(\mathcal{H})^{+}$.

Proof. Decompose $\mathcal{H}$ into an orthogonal sum of two isomorphic closed subspaces, $\mathcal{H}=\mathcal{K} \oplus \mathcal{K}$; then $T$ is represented by an operator matrix of the form

$$
T=\left[\begin{array}{cc}
A & B \\
C & D
\end{array}\right]
$$

First we will try to find diagonal positive operators $T_{j}=a_{j} \oplus b_{j}\left(a_{j}, b_{j} \in \mathrm{B}(\mathcal{K})\right)$ and invertible operators $S_{j}(j=1, \ldots, 4)$ of the form (2.1) such that $T=\sum_{j=1}^{4} S_{j} T_{j} S_{j}^{-1}$. It turns out that we can even take $S_{j}$ of the form

$$
S_{j}=\left[\begin{array}{cc}
1 & x_{j} \\
y_{j} & 1+y_{j} x_{j}
\end{array}\right] .
$$

Then

$$
S_{j} T_{j} S_{j}^{-1}=\left[\begin{array}{cc}
a_{j}+s_{j} y_{j} & -s_{j} \\
y_{j} a_{j}-b_{j} y_{j}+y_{j} s_{j} y_{j} & b_{j}-y_{j} s_{j}
\end{array}\right], \text { where } s_{j}:=a_{j} x_{j}-x_{j} b_{j} .
$$

There are many appropriate choices for $x_{j}, y_{j}, z_{j}, a_{j}, b_{j}$ in order to make the sum $\sum_{j=1}^{4} S_{j} T_{j} S_{j}^{-1}$ equal to $T$. For example, if we let $y_{1}=0=x_{2}, y_{3}=1, b_{1}=0$ and for $j \geq 2$ choose all $a_{j}$ and $b_{j}$ to be positive scalars with $a_{j}-b_{j}=1$, and denote $\beta=\sum_{j=2}^{4} b_{j}$ (so that $\sum_{j=2}^{4} a_{j}=\beta+3$ ), then

$$
\sum_{j=1}^{4} S_{j} T_{j} S_{j}^{-1}=\left[\begin{array}{cc}
a_{1}+\beta+3+x_{3}+x_{4} y_{4} & -a_{1} x_{1}-x_{3}-x_{4} \\
y_{2}+x_{3}+1+y_{4}+y_{4} x_{4} y_{4} & \beta-x_{3}-y_{4} x_{4}
\end{array}\right] .
$$

To achieve that the matrix in (2.4) will be equal to $T$, we only need to choose $x_{3}, x_{4}, y_{4}$ in $\mathrm{B}(\mathcal{K})$ and invertible $a_{1} \in \mathrm{B}(\mathcal{K})^{+}$so that

$$
a_{1}+\beta+3+x_{3}+x_{4} y_{4}=A \text { and } \beta-x_{3}-y_{4} x_{4}=D,
$$

for then the off-diagonal terms of the matrix (2.4) can be made equal to $B$ and $C$ by a suitable choice of $y_{2}$ and $x_{1}$. Adding the two equations (2.5), we see that we only need to choose $x_{4}, y_{4}$ and $a_{1}$ so that

$$
x_{4} y_{4}-y_{4} x_{4}=A+D-a_{1}-2 \beta-3=: T_{0},
$$

for then $x_{3}$ can be computed from either of the equations (2.5). So (for a fixed $\beta$ ), we first choose an invertible positive $a_{1} \in \mathrm{B}(\mathcal{K})$ of the form $\lambda+\mu p$, where $\lambda, \mu \in \mathbb{R}^{+}$and $p$ is a projection of infinite rank and nullity, such that $\sigma\left(a_{1}\right) \subset(0,1]$ and $T_{0}$ is not a compact perturbation of a scalar. Then $T_{0}$ is a commutator by [2] (a simplified proof is in [1]), which means that there exist $x_{4}$ and $y_{4}$ satisfying 
(2.6). By suitably choosing scalars $a_{j}$ and $b_{j}(j \geq 2)$ we can make the spectra of $T_{j}$ disjoint for all $j$.

Remark 2.2. For a later use observe that in the above proof the spectra of $a_{j}$ and $b_{j}$ are disjoint for all $j$; in fact, all $a_{j}$ and $b_{j}$ chosen above are scalars, except possibly $a_{1}$. Also note that the operator $S_{1} T_{1} S_{1}^{-1}$ has the form

$$
\left[\begin{array}{cc}
a_{1} & * \\
0 & 0
\end{array}\right]
$$

where $a_{1} \in \mathrm{B}(\mathcal{K})^{+}$.

Theorem 2.3. Every $T \in \mathrm{B}(\mathcal{H})$ is of the form $T=\sum_{j=1}^{3} S_{j} T_{j} S_{j}^{-1}$, where $S_{j} \in$ $\mathrm{B}(\mathcal{H})$ and the operators $T_{j} \in \mathrm{B}(\mathcal{H})$ are positive (and invertible for $j \leq 2$ ) with finite spectra $\sigma\left(T_{j}\right)$, amd each $\sigma\left(T_{j}\right)$ consists of at most four points. Moreover, 0 is an isolated point of $\sigma\left(T_{3}\right)$, and the range of $T_{3}$ is closed and has infinite dimension and codimension.

Proof. As in the proof of Lemma 2.1 we represent $T$ by the operator matrix (2.3). Now we try to find positive block-diagonal operators $T_{j}=a_{j} \oplus b_{j}$ and invertible operators $S_{j} \in \mathrm{B}(\mathcal{H})$ of the form (2.1) (with $z-y u^{-1} x=1$ ) such that $\sum_{j=1}^{3} S_{j} T_{j} S_{j}^{-1}=T$. Denoting

$$
S_{j}=\left[\begin{array}{ll}
u_{j} & x_{j} \\
y_{j} & z_{j}
\end{array}\right], \text { where } u_{j} \text { is invertible and } z_{j}-y_{j} u_{j}^{-1} x_{j}=1,
$$

we compute (using (2.2) ) that

$$
S_{j} T_{j} S_{j}^{-1}=\left[\begin{array}{cc}
c_{j}+s_{j} v_{j} & -s_{j} \\
v_{j} c_{j}-b_{j} v_{j}+v_{j} s_{j} v_{j} & b_{j}-v_{j} s_{j}
\end{array}\right],
$$

where

$$
c_{j}:=u_{j} a_{j} u_{j}^{-1}, \quad v_{j}:=y_{j} u_{j}^{-1}, \text { and } s_{j}:=c_{j} x_{j}-x_{j} b_{j} .
$$

Note that if the spectra of $b_{j}$ and $c_{j}$ are disjoint, then from (2.7) $a_{j}, y_{j}, b_{j}$ and $x_{j}$ can all be computed from $c_{j}, u_{j}, v_{j}, b_{j}$, and $s_{j}$. (That the equation $c_{j} x_{j}-x_{j} b_{j}=s_{j}$ can be solved for $x_{j}$ is Rosenblum's theorem [14, p. 8].) Further, we assume that the matrix $S_{3}$ is diagonal (that is, $x_{3}=0=y_{3}$, so we will only need that the spectra of $c_{j}$ and $b_{j}$ are disjoint for $\left.j=1,2\right)$. Then the condition $\sum S_{j} T_{j} S_{j}^{-1}=T$ is equivalent to the following four equations:

$$
\begin{gathered}
s_{1} v_{1}+s_{2} v_{2}=A-c_{1}-c_{2}-c_{3}, \quad s_{1}+s_{2}=-B, \\
v_{1} c_{1}-b_{1} v_{1}+v_{2} c_{2}-b_{2} v_{2}+v_{1} s_{1} v_{1}+v_{2} s_{2} v_{2}=C, \\
v_{1} s_{1}+v_{2} s_{2}=-D+b_{1}+b_{2}+b_{3} .
\end{gathered}
$$

Set $c:=c_{1}+c_{2}+c_{3}, b:=b_{1}+b_{2}+b_{3}$ and

$$
s:=s_{1}, \quad v:=v_{2}, \quad w:=v_{2}-v_{1} .
$$

Then from the second equation in (2.8) we get $s_{2}=-(B+s)$; using this, the other three equations (2.8), (2.9) can be rewritten as

$$
\begin{gathered}
B v+s w=c-A, \quad v B+w s=D-b, \\
v\left(c_{1}+c_{2}-s w\right)-\left(b_{1}+b_{2}+w s\right) v-w c_{1}+b_{1} w+w s w-v B v=C .
\end{gathered}
$$


From (2.10) we have that $c_{1}+c_{2}-s w=A-c_{3}+B v$ and $b_{1}+b_{2}+w s=D-b_{3}-v B$; hence (2.11) can be rewritten as

$$
w s w-w c_{1}+b_{1} w=C-v\left(A-c_{3}\right)+\left(D-b_{3}\right) v-v B v .
$$

We are going to show that the system of equations (2.10), (2.12) has a solution.

First suppose that $T$ is not a compact perturbation of a scalar. Then we may assume that in the matrix representation of $T$ we have that $D=0$ and that $B$ is an isometry with the range of $B$ isomorphic to its orthogonal complement in $\mathcal{K}$ since by [2, Corollary 3.4] $T$ is similar to such an operator. In this case we shall see that we can even afford to choose $s=0$, so that the above system of equations simplifies to

$$
\begin{gathered}
B v=c-A, \\
v B=-b, \\
b_{1} w-w c_{1}=C-v\left(A-c_{3}\right)+\left(-b_{3}\right) v-v B v .
\end{gathered}
$$

Since $B^{*} B=1$, the equation (2.13) is equivalent to the following two:

$$
v=B^{*}(c-A) \text { and } P^{\perp}(c-A)=0 \text {, where } P:=B B^{*} \text { and } P^{\perp}:=1-P .
$$

Using this expression for $v$, (2.14) can be rewritten as

$$
b_{1}+b_{2}+b_{3}=b=B^{*}(A-c) B .
$$

If there exist $v, c_{j}$ and $b_{j}(j=1,2,3)$ such that the equations (2.16) and (2.17) are satisfied and the spectra of $c_{1}$ and $b_{1}$ are disjoint, then the equation (2.15) can be solved for $w$ by Rosenblum's theorem.

To show that the system (2.16), (2.17) has a solution, represent $A$ by a $2 \times 2$ operator matrix with respect to the decomposition $\mathcal{K}=P \mathcal{K} \oplus P^{\perp} \mathcal{K}$. By Lemma 2.1, $A=\sum_{j=1}^{4} A_{j}$, where each $A_{j}$ is similar to a positive operator; moreover, by Remark 2.2 we may assume that (with respect to the decomposition $\mathcal{K}=P \mathcal{K} \oplus P^{\perp} \mathcal{K}$ ) $A_{4}$ is of the form

$$
A_{4}=\left[\begin{array}{ll}
a & r \\
0 & 0
\end{array}\right], \text { where } a \geq 0,
$$

which means that $P^{\perp} A_{4}=0$. Thus, if we put $c_{j}=A_{j}$ for $j=1,2,3$ (and $c=$ $\left.c_{1}+c_{2}+c_{3}\right)$, then we have $P^{\perp}(A-c)=P^{\perp} A_{4}=0$, which is just the condition in (2.16). Further

$$
B^{*}(A-c) B=B^{*} A_{4} B=B^{*} A_{4} P B=B^{*} G B
$$

where

$$
G:=A_{4} P=a \oplus 0 .
$$

Thus the operator $B^{*}(A-c) B$ is positive and hence it can be written (in many ways) as a sum of three positive operators $b_{j}$, which is just what the condition (2.17) requires. We may choose $b_{3}=0$. To see that it is possible to choose $b_{j}$ and $c_{j}(j=1,2)$ so that their spectra are disjoint, note that $P B$ is a unitary operator from $\mathcal{K}$ onto $P \mathcal{K}$ which intertwines $b$ and $a$ by (2.19) and (2.17); hence $b$ and $a$ have the same spectrum. By Lemma 2.1 we may choose $a$ and $c_{j}=A_{j}$ so that each of their spectra consists of at most two points, $\sigma(a) \subseteq(0,1]$ and $\sigma\left(A_{j}\right) \subset(1, \infty)$ $(j=1,2,3)$. Since $b_{j} \geq 0$ and $b_{1}+b_{2}=b$, the spectra of $b_{j}$ are contained in $[0,1]$; hence $\sigma\left(b_{j}\right) \cap \sigma\left(c_{j}\right)=\emptyset$. Since $\sigma(b)$ consists of at most two points in $(0,1]$, we may 
choose $b_{1}, b_{2}$ to have the same property. (We may choose for $b_{1}$ a sufficiently small positive scalar, for example.)

Since $T_{j}$ is similar to $a_{j} \oplus b_{j}$ and $a_{j}$ is similar to $c_{j}=A_{j}(j=1,2,3), \sigma\left(T_{j}\right)=$ $\sigma\left(A_{j}\right) \cup \sigma\left(b_{j}\right)$ consists of at most four points. Other properties of the operators $T_{j}$ stated in the theorem also follow easily from those of $c_{j}$ and $a_{j}$ chosen above.

Now we consider the case when $T$ is a compact perturbation of a scalar. In this case let $E=1 \oplus 0$, the projection onto the first summand in the decomposition $\mathcal{H}=\mathcal{K} \oplus \mathcal{K}$. Then $\tilde{T}:=T-E$ is not a compact perturbation of a scalar, so by the already proved case $\tilde{T}$ can be expressed as $\tilde{T}=\sum_{j=1}^{3} S_{j}\left(a_{j} \oplus b_{j}\right) S_{j}^{-1}$, where $a_{j} \geq 0$ and $b_{j} \geq 0$ and $S_{3}$ is block-diagonal. Since $S_{3}$ commutes with $E$, we have

$$
T=\tilde{T}+E=\sum_{j=1}^{2} S_{j}\left(a_{j} \oplus b_{j}\right) S_{j}^{-1}+S_{3}\left(\left(a_{3} \oplus b_{3}\right)+E\right) S_{3}^{-1},
$$

which is a sum of three operators similar to positive ones with (at most) four-point spectra.

Remark 2.4. Observe that in the proof of Theorem 2.3 the operator $T_{3}$ is of the form $e \oplus 0$ (since $b_{3}=0$ and $S_{3}$ is a diagonal $2 \times 2$ operator matrix), where $e$ is similar to a positive invertible operator with at most two-point spectrum.

Corollary 2.5. Each $T \in \mathrm{B}(\mathcal{H})$ can be expressed as $T=\sum_{j=1}^{3} A_{j} B_{j}$, where $A_{j}, B_{j} \in \mathrm{B}(\mathcal{H})^{+}$.

Theorem 2.6. If $T \in \mathrm{B}(\mathcal{H})$ is not a compact perturbation of a scalar, then $T$ is a sum of two operators similar to positive operators.

Proof. We have to show that in the proof of Theorem 2.3, $a_{3}$ and $b_{3}$ can be taken to be 0 . That $b_{3}$ can be taken to be 0 has been already observed in that proof. Now note that in the matrix representation (2.3) of $T$ we may assume, in addition to $D=0$ and $B$ is an isometry, that $A$ is not a compact perturbation of a scalar. For this, we simply decompose the second copy of $\mathcal{K}$ into two orthogonal isomorphic closed subspaces, $\mathcal{K}=\mathcal{K}_{0} \oplus \mathcal{K}_{1}$, and decompose $\mathcal{H}$ as $\mathcal{H}=\mathcal{K}_{1}^{\perp} \oplus \mathcal{K}_{1}$. Since $B$ maps $\mathcal{K}_{1}$ isometrically into $\mathcal{K}_{1}^{\perp}$ the matrix of $T$ has 0 in the $(2,2)$ position and an isometry with infinitely codimensional range in the $(1,2)$ position. The new element in the position $(1,1)$ is then not a compact perturbation of a scalar. So we will assume that already in the initial matrix representation of $T$, the element $A$ is not a compact perturbation of a scalar. Now consider the matrix of $A$ relative to the decomposition of the Hilbert space of $A$ into the range of $B$ and its orthogonal complement. Since $A$ is not a compact perturbation of a scalar, by Theorem 2.3 and Remark 2.4. $A$ is of the form $A=\sum_{j=1}^{3} \tilde{A}_{j}$, where $\tilde{A}_{1}$ and $\tilde{A}_{2}$ are similar to positive invertible operators each with at most four points in its spectrum and $\tilde{A}_{3}$ is of the form $e \oplus 0$ with $e$ similar to a positive invertible operator with a twopoint spectrum. By the same reasoning as in the proof of Theorem 2.3 (see the paragraph containing (2.18); the role of $A_{4}$ is now played by $\tilde{A}_{3}$ ) we see that the system of equations (2.16), (2.17) has a solution such that $c_{j}=\tilde{A}_{j}$ for $j=1,2$ and $c_{3}=0=b_{3}=0$. But we have to show also that we can achieve $\sigma\left(c_{j}\right) \cap \sigma\left(b_{j}\right)=\emptyset$ $(j=1,2)$ in order to assure that (2.15) has a solution for $w$ and that $x_{j}$ can be computed from the last equation in (2.7). For this we now note that the operator $B^{*}(A-c) B=B^{*} \tilde{A}_{3} B$ is unitarily equivalent to $e$. Since $\sigma\left(c_{j}\right)(j=1,2)$ is a finite subset of $(0, \infty)$ and $\sigma\left(B^{*}(A-c) B\right)$ consists of just two positive points, it follows 
that $B^{*}(A-c) B$ is similar to a sum $b_{1}+b_{2}$, where $b_{j} \geq 0$ and $\sigma\left(b_{j}\right) \cap \sigma\left(c_{j}\right)=\emptyset$ for both $j=1,2$.

An operator $T \in \mathrm{B}(\mathcal{H})$ of the form $\lambda+K$, where $\lambda \in \mathbb{C} \backslash \mathbb{R}^{+}$and $K$ is compact, is not of the form

$$
P Q+R S \text { for any } P, Q, R, S \in \mathrm{B}(\mathcal{H})^{+} .
$$

To see this, just note that the spectrum of the coset $\dot{R} \dot{S}$ in the Calkin algebra is the same as the spectrum of $\dot{S}^{1 / 2} \dot{R} \dot{S}^{1 / 2}$, hence contained in $\mathbb{R}^{+}$, while the spectrum of $\lambda-\dot{P} \dot{Q}$ is contained in the ray $\lambda-\mathbb{R}^{+}$which is disjoint with $\mathbb{R}^{+}$.

Each compact operator on a Hilbert space is an additive commutator of two bounded operators [1. By an analogy one might conjecture that each compact operator is a sum of two operators similar to positive ones, but this is not true.

Proposition 2.7. If $T \in \mathrm{C}^{1}(\mathcal{H})$ (the trace class) is nonzero and $\operatorname{Tr}(T)$ is not positive, then $T$ is not a sum of two operators in $\mathrm{B}(\mathcal{H})$ similar to positive ones.

Proof. Assume the contrary, that $T=S_{1} A S_{1}^{-1}+S_{2} B S_{2}^{-1}$, where $A, B \in \mathrm{B}(\mathcal{H})^{+}$. Put $F:=-S_{1}^{-1} T S_{1}$ and $S=S_{1}^{-1} S_{2}$. Then

$$
F+A=-S B S^{-1} \text {. }
$$

Considering the essential spectra, it follows from (2.21) and the positivity of $A$ and $B$ that $A$ and $B$ must be compact. We claim that $A$ and $B$ must be in the Hilbert-Schmidt class $\mathrm{C}^{2}(\mathcal{H})$. For a proof we may first replace $B$ by a unitarily equivalent operator (and modify $S$ accordingly) to reduce to the situation when $A$ and $B$ can be diagonalized in the same orthonormal basis $\mathbb{B}$ of $\mathcal{H}$. Let $\left(\alpha_{j}\right)$ and $\left(\beta_{j}\right)$ be the lists of eigenvalues of $A$ and $B$ in decreasing order (each eigenvalue repeated according to its multiplicity). From (2.21) we have $A S+S B=G$, where $G:=-F S$. Denoting by $\sigma_{i, j}$ and $\psi_{i, j}$ the entries of the matrices of $S$ and $G$ in the basis $\mathbb{B}$, we see that

$$
\left(\alpha_{i}+\beta_{j}\right) \sigma_{i, j}=\psi_{i, j}
$$

Let $\gamma_{j}:=\left(\sum_{i}\left|\psi_{i, j}\right|^{2}\right)^{1 / 2}$ and note that $\sum_{j} \gamma_{j}^{2}<\infty$ since $G \in \mathrm{C}^{2}(\mathcal{H})$. Since $S$ is invertible (in particular, bounded from below), there exists a scalar $\gamma>0$ such that $\sum_{i}\left|\sigma_{i, j}\right|^{2} \geq \gamma$ for all $i$; hence (2.22) implies that

$$
\beta_{j}^{-2} \gamma_{j}^{2}=\beta_{j}^{-2} \sum_{i}\left|\psi_{i, j}\right|^{2}=\sum_{i} \frac{\left(\alpha_{i}+\beta_{j}\right)^{2}}{\beta_{j}^{2}}\left|\sigma_{i, j}\right|^{2} \geq \sum_{i}\left|\sigma_{i, j}\right|^{2} \geq \gamma,
$$

whenever $\beta_{j} \neq 0$. Thus $\beta_{j}^{2} \leq \gamma_{j}^{2} \gamma^{-1}$ and consequently $\sum_{j} \beta_{j}^{2}<\infty$, which means that $B \in \mathrm{C}^{2}(\mathcal{H})$. Similarly (or from (2.21), since $F \in \mathrm{C}^{2}(\mathcal{H})$ ) we see that $A \in$ $\mathrm{C}^{2}(\mathcal{H})$.

By considering the polar decomposition of $S$ of the form $S=R U$, where $R$ is positive and $U$ is unitary, we may rewrite (2.21) in the form

$$
F+A=-R C R^{-1}
$$

where $C:=U B U^{*} \geq 0$. Assume for a moment that in some orthonormal basis of $\mathcal{H}$ the operator $R$ can be represented by a diagonal matrix and let $\left[\alpha_{i, j}\right],\left[\phi_{i, j}\right]$ and 
$\left[\gamma_{i, j}\right]$ be the matrices of $A, F$ and $C$ (respectively) in this basis. Then, considering the sums of diagonal terms of matrices, (2.23) implies that

$$
-\sum_{j=1}^{n} \phi_{j, j}=\sum_{j=1}^{n} \alpha_{j, j}+\sum_{j=1}^{n} \gamma_{j, j} .
$$

Letting $n \rightarrow \infty$, the first sum in (2.24) tends to $-\operatorname{Tr}(F)=\operatorname{Tr}(T) \in \mathbb{C} \backslash(0, \infty)$, while the second and the third sums converge to elements in $[0, \infty]$. This shows that the equality (2.24) can hold for all $n$ only if $\operatorname{Tr}(T)=0$ and $\phi_{j, j}=0=\alpha_{j, j}$ for all $j$. Since $A \in \mathrm{B}(\mathcal{H})^{+}$, the condition $\alpha_{j, j}=0$ for all $j$ implies that $A=0$. But then $B$ is similar to $T$; hence $\operatorname{Tr}(B)=0$, which implies (since $B \geq 0$ ) that $B=0$. In this case $T=0$, which was excluded by the hypothesis of the proposition. Now we will show by an approximation argument that (2.23) leads to a contradiction even if $R$ cannot be diagonalized.

By the Weyl-von Neumann theorem [4, p. 214], given $\varepsilon>0$, there exist a diagonal Hermitian operator $D$ and an operator $H \in \mathrm{C}^{2}(\mathcal{H})$ with $\|H\|_{2}<\varepsilon$ (where $\|\cdot\|_{2}$ denotes the Hilbert-Schmidt norm) such that $R=D+H$. If $\varepsilon$ is small enough, then $D$ is invertible (since $D=R-H=R\left(1-R^{-1} H\right)$ ) and

$$
\left\|D^{-1}\right\| \leq\left\|R^{-1}\right\| \sum_{n=0}^{\infty}\left\|R^{-1} H\right\|^{n} \leq \frac{\left\|R^{-1}\right\|}{1-\varepsilon\left\|R^{-1}\right\|} .
$$

Further, if $\varepsilon$ is small enough, then $1+H D^{-1}$ is invertible and

$$
R C R^{-1}=\left(1+H D^{-1}\right) D C D^{-1}\left(1+H D^{-1}\right)^{-1} .
$$

Since $\left(1+H D^{-1}\right)^{-1}=1-H D^{-1}\left(1+H D^{-1}\right)^{-1}$, we may write

$$
\begin{aligned}
R C R^{-1}=D C D^{-1}-D C D^{-1} H D^{-1}(1 & \left.+H D^{-1}\right)^{-1} \\
& +H C D^{-1}\left[1-H D^{-1}\left(1+H D^{-1}\right)^{-1}\right] ;
\end{aligned}
$$

hence (since $B$ and therefore also $C$ is in $\mathrm{C}^{2}(\mathcal{H})$ by the first paragraph of this proof)

$$
\begin{aligned}
& \left\|R C R^{-1}-D C D^{-1}\right\|_{1} \leq\|H\|_{2}\|C\|_{2}\left\|D^{-1}\right\| \\
& \cdot\left[\|D\|\left\|D^{-1}\right\|\left\|\left(1+H D^{-1}\right)^{-1}\right\|+\left\|1-H D^{-1}\left(1+H D^{-1}\right)^{-1}\right\|\right] .
\end{aligned}
$$

It follows that $\left\|R C R^{-1}-D C D^{-1}\right\|_{1} \rightarrow 0$ as $\varepsilon \rightarrow 0$. This allows us to conclude in essentially the same way as in the previous paragraph (by considering the sums of diagonal entries of matrices) that (2.23) leads to a contradiction.

For most of the above proof it would be sufficient if we assumed that $T \in \mathrm{C}^{2}(\mathcal{H})$ (instead of $T \in \mathrm{C}^{1}(\mathcal{H})$ ), but the problem is that for an operator $T$ not in $\mathrm{C}^{1}(\mathcal{H})$ the sum of diagonal entries of its matrix relative to a general orthogonal basis can be quite arbitrary (it need not even be defined [7]).

Problem. Which compact operators on an infinite dimensional Hilbert space can be written as $T_{1}+T_{2}$, where $T_{1}$ and $T_{2}$ are similar to positive operators?

Theorem 2.6 implies that all operators can be approximated in norm by sums of two operators similar to positive ones, but concerning such an approximation a much stronger result holds: it follows from [6, Theorem 3.10] that both summands can be taken to be similar to the same positive operator. 


\section{ON SPECTRA OF LÜDERS OPERATORS}

For two commutative $m$-tuples $\left(A_{j}\right)$ and $\left(B_{j}\right)$ of elements of $\mathrm{B}(\mathcal{H})$ the spectrum $\sigma(\Phi)$ of the map $\Phi(X):=\sum_{j=1}^{m} A_{j} X B_{j}$ on $\mathrm{B}(\mathcal{H})$ can be described in terms of spectra of $\left(A_{j}\right)$ and $\left(B_{j}\right)$ ([5], [1] $)$; in particular, $\sigma(\Phi) \subseteq \mathbb{R}^{+}$if $A_{j}, B_{j} \in \mathrm{B}(\mathcal{H})^{+}$. For noncommutative $\left(A_{j}\right)$ and $\left(B_{j}\right)$ the situation may be completely different. One consequence of Theorem 2.3 is that for an infinite dimensional Hilbert space $\mathcal{H}$ the spectra of Lüders operators on $\mathrm{B}(\mathcal{H})$ are not necessarily contained in $\mathbb{R}^{+}$.

Proposition 3.1. Let $\mathcal{H}$ be an infinite dimensional Hilbert space. Every complex number $\lambda$ can be an eigenvalue of a Lüders operator on $\mathrm{B}(\mathcal{H})$ of length 3 (or more).

Proof. Decompose $\mathcal{H}$ as $\mathcal{H}=\mathcal{K} \oplus \mathcal{K}$. By Corollary 2.5 there exist $A_{j}, B_{j} \in \mathrm{B}(\mathcal{K})^{+}$ such that $\sum_{j=1}^{3} A_{j} B_{j}=\lambda$. By a simple calculation this implies that the operator

$$
X_{0}:=\left[\begin{array}{ll}
0 & 1 \\
0 & 0
\end{array}\right]
$$

is an eigenvector corresponding to the eigenvalue $\lambda$ of the Lüders operator $\Phi$ on $\mathrm{B}(\mathcal{H})$ defined by $\Phi(X)=\sum_{j=1}^{3} T_{j} X T_{j}$, where

$$
T_{j}=\left[\begin{array}{cc}
A_{j} & 0 \\
0 & B_{j}
\end{array}\right] .
$$

Theorem 3.2. Suppose that $A_{j}, B_{j} \in \mathrm{B}(\mathcal{H})^{+}(j=1,2)$ and let $\Phi$ be the map on $\mathrm{B}(\mathcal{H})$ defined by $\Phi(X)=\sum_{j=1}^{2} A_{j} X B_{j}$. If $A_{1} A_{2}=A_{2} A_{1}$ (or if $B_{1} B_{2}=B_{2} B_{1}$ ), then the spectrum of $\Phi$ is contained in $\mathbb{R}^{+}$.

Proof. Since boundary points of the spectrum of any operator are approximate eigenvalues [3], it suffices to show that each approximate eigenvalue $\lambda$ of $\Phi$ is in $\mathbb{R}^{+}$. By considering the space $\mathcal{B}:=\ell_{\infty}(\mathrm{B}(\mathcal{H})) / c_{0}(\mathrm{~B}(\mathcal{H}))$, where $\ell_{\infty}(\mathrm{B}(\mathcal{H}))$ is the space of all bounded sequences with entries in $\mathrm{B}(\mathcal{H})$, and $c_{0}(\mathrm{~B}(\mathcal{H}))$ is the subspace of all sequences converging (in norm) to 0 , we may reduce the approximate eigenvalues of $\Phi$ to proper eigenvalues of the corresponding operator $\tilde{\Phi}$ on $\mathcal{B}$. Here of course $\tilde{\Phi}$ is defined by $\tilde{\Phi}\left(\left[X_{n}\right]\right)=\left[\Phi\left(X_{n}\right)\right]$, where $\left[X_{n}\right]$ denotes the coset of a sequence $\left(X_{n}\right) \in \ell_{\infty}(\mathrm{B}(\mathcal{H}))$. Note that $\tilde{\Phi}$ is again an elementary operator, namely of the form

$$
\tilde{\Phi}(Y)=\sum_{j=1}^{2} \tilde{A}_{j} Y \tilde{B}_{j} \quad(Y \in B),
$$

where $\tilde{A}$ denotes the coset in $\mathcal{B}$ of the constant sequence $(A, A, \ldots) \in \ell_{\infty}(\mathrm{B}(\mathcal{H}))$ for each $A \in \mathrm{B}(\mathcal{H})$. Since $\mathcal{B}$ is a $C^{*}$-algebra, we can regard it as a subalgebra of $\mathrm{B}(\mathcal{K})$ for some (nonseparable) Hilbert space $\mathcal{K}$, and by the formula (3.1) we may regard the map $\tilde{\Phi}$ to be defined on $\mathrm{B}(\mathcal{K})$. Any approximate eigenvalue $\lambda$ of $\Phi$ is then an eigenvalue of $\tilde{\Phi}$. Choose a nonzero eigenvector $Y$ corresponding to $\lambda$. $\mathcal{K}$ is not separable, but it can be expressed as an orthogonal sum of separable subspaces $\mathcal{K}_{i}$ that reduce all the operators $A_{j}, B_{j}$ and $Y$. If $i$ is such that $Y \mid \mathcal{K}_{i} \neq 0$, then $\lambda$ is an eigenvalue of the operator $\Psi$ on $\mathrm{B}\left(\mathcal{K}_{i}\right)$ defined by $\Psi(X)=\sum_{j=1}^{2} C_{j} X D_{j}$, where $C_{j}=A_{j} \mid \mathcal{K}_{i}$ and $D_{j}=B_{j} \mid \mathcal{K}_{i}$. So it suffices to show that all eigenvalues of 
such operators are in $\mathbb{R}^{+}$. Thus (adapting the notation), we may assume that $\lambda$ is an eigenvalue of $\Phi$. Denote by $X$ a corresponding eigenvector with $\|X\|=1$; hence

$$
\sum_{j=1}^{2} A_{j} X B_{j}=\lambda X
$$

Suppose that $A_{1}$ and $A_{2}$ commute. Then by Voiculescu's version [17 of the Weyl-von Neumann-Berg theorem, given $\varepsilon>0$, there exist commuting diagonal Hermitian operators $C_{j} \in \mathrm{B}(\mathcal{H})$ and Hilbert-Schmidt operators $H_{j} \in \mathrm{C}^{2}(\mathcal{H})$ such that $A_{j}=C_{j}+H_{j}$ and $\left\|H_{j}\right\|_{2}<\varepsilon(j=1,2)$. Let $C_{j}=C_{j}^{+}-C_{j}^{-}$be the decomposition of $C$ into the positive and the negative part and denote by $Q_{j}$ the range projection of $C_{j}^{-}$. Then $A_{j}+C_{j}^{-}=C_{j}^{+}+H_{j}$; hence (since $Q_{j} C_{j}^{+}=0$ and $\left.Q_{j} C_{j}^{-}=C_{j}^{-}\right)$

$$
Q_{j} A_{j} Q_{j}+C_{j}^{-}=Q_{j} H_{j} Q_{j} \in \mathrm{C}^{2}(\mathcal{H}) .
$$

This implies that $C_{j}^{-} \in \mathrm{C}^{2}(\mathcal{H})$ and $\left\|C_{j}^{-}\right\|_{2} \leq\left\|H_{j}\right\|_{2}<\varepsilon$. So, replacing $C_{j}$ by $C_{j}^{+}$ and $H_{j}$ by $H_{j}-C_{j}^{-}$(and the initial $\varepsilon$ by $\varepsilon / 2$ ), we may assume that $C_{j} \geq 0$. Let $P$ be any finite rank projection that commutes with $C_{1}$ and $C_{2}$. (Note that, since $C_{1}, C_{2}$ are commuting diagonal operators, there exists a net of such projections $P$ converging strongly to the identity.) From (3.2) we have that $\sum P A_{j} X B_{j} X^{*} P=$ $\lambda P X X^{*} P$; hence applying the trace $\operatorname{Tr}$ we obtain

$$
\sum_{j=1}^{2}\left(\operatorname{Tr}\left(P C_{j} X B_{j} X^{*} P\right)+\operatorname{Tr}\left(P H_{j} X B_{j} X^{*} P\right)\right)=\lambda \operatorname{Tr}\left(P X X^{*} P\right) .
$$

Since $P$ commutes with $C_{j}$,

$$
\operatorname{Tr}\left(P C_{j} X B_{j} X^{*} P\right)=\operatorname{Tr}\left(C_{j} P X B_{j} X^{*} P\right)=\operatorname{Tr}\left(C_{j}^{1 / 2} P X B_{j} X^{*} P C_{j}^{1 / 2}\right) \geq 0 .
$$

Further (since $\|Z\|_{2}=\left\|Z^{*}\right\|_{2}$ for all $Z \in \mathrm{B}(\mathcal{H})$ ),

$$
\mid \operatorname{Tr}\left(P H_{j} X B_{j} X^{*} P \mid \leq\left\|H_{j}\right\|_{2}\left\|X B_{j} X^{*} P\right\|_{2}=\left\|H_{j}\right\|_{2}\left\|P X B_{j} X^{*}\right\|_{2}<\varepsilon\|P X\|_{2},\right.
$$

where we have assumed (without lost of generality) that $\left\|B_{j}\right\| \leq 1$. If $P$ is sufficiently close to 1 so that $P X \neq 0$, then from (3.3) and (3.5) we have that

$$
\begin{aligned}
\left|\lambda-\sum_{j=1}^{2} \frac{\operatorname{Tr}\left(P C_{j} X B_{j} X^{*} P\right)}{\operatorname{Tr}\left(P X X^{*} P\right)}\right| & \leq \varepsilon \sum_{j=1}^{2} \frac{\|P X\|_{2}}{\operatorname{Tr}\left(P X X^{*} P\right)} \\
& =\frac{2 \varepsilon}{\|P X\|_{2}} .
\end{aligned}
$$

Letting in this estimate $P \rightarrow 1, \varepsilon \rightarrow 0$ and using (3.4), we see that $\lambda \geq 0$.

Remark 3.3. Theorem 3.2 can be extended to operators of the form

$$
X \mapsto \sum_{j=1}^{n} A_{j} X B_{j}
$$

if the coefficients on one side, say all the $A_{j}$, are smooth nonnegative functions $A_{j}=f_{j}\left(H_{1}, H_{2}\right)$ of a pair of commuting Hermitian operators $\left(H_{1}, H_{2}\right)$. Namely, in this case it can be shown (using the Fourier transform) that small HilbertSchmidt perturbations of $\left(H_{1}, H_{2}\right)$ result in small Hilbert-Schmidt perturbations 
of $f_{j}\left(H_{1}, H_{2}\right)$. The author does not know if the theorem can be extended to the general situation, when all the $A_{j}$ commute, but the $B_{j}$ do not necessarily commute.

Problems. 1. Can Theorem 3.2 be generalized to operators of length greater than $2 ?$

2. Suppose that all $A_{j}, B_{j}$ are positive and for each $j$ at least one of $A_{j}, B_{j}$ is compact. Then it can be deduced from [15. Corollary 6.6] (see [10]) that all eigenvalues of the operator (3.6) are contained in $\mathbb{R}^{+}$. Is the same true for the entire spectrum?

3. In Theorem 3.2 can the commutativity condition be replaced by commutativity modulo compact operators?

\section{ACKNOWLEDGMENTS}

The author is very grateful to Professor Heydar Radjavi for the discussion concerning sums of projections and positive operators. He is also grateful to Roman Drnovšek for bringing reference [19] to his attention.

\section{REFERENCES}

[1] J. H. Anderson and J. G. Stampfli, Commutators and compressions, Israel J. Math. 10 (1971), 433-441. MR0312312 (47:874)

[2] A. Brown and C. Pearcy, Structure of commutators of operators, Ann. Math. 82 (1965), 112-127. MR0178354(31:2612)

[3] J. B. Conway, A course in functional analysis, GTM 96, Springer, Berlin, 1985. MR768926 (86h:46001)

[4] J. B. Conway, A course in operator theory, GSM 21, Amer. Math. Soc., Providence, RI, 2000. MR 1721402 (2001d:47001)

[5] R. E. Curto, Spectral theory of elementary operators, Elementary Operators and Appl. (M. Mathieu, editor), 3-52, World Scientific, Singapore, 1992. MR.1183936 (93i:47041)

[6] K. R. Davidson and L. W. Marcoux, Linear spans of unitary and similarity orbits of a Hilbert space operator, J. Operator Theory 52 (2004), 113-132. MR2091463 (2005e:47048)

[7] P. Fan, C. K. Fong and D. Herrero, On zero-diagonal operators and traces, Proc. Amer. Math. Soc. 99 (1987), 445-451. MR875378 (88c:47009)

[8] D. W. Kribs, A quantum computing primer for operator theorists, Linear Algebra Appl. 400 (2005), 147-167. MR2131922(2005k:47170)

[9] L. Long and S. Zhang, Fixed points of commutative super-operators, J. Phys. A: Math. Theor. 44 (2011), 095201. MR2771865

[10] B. Magajna, Fixed points of normal completely positive maps on B(H), J. Math. Anal. Appl. 389 (2012), 1291-1302. MR2879297

[11] G. Nagy, On spectra of Lüders operations, J. Math. Phys. 49 (2008), no. 2, 022110. MR.2392847(2009b:81086)

[12] C. Pearcy and D. Topping, Sums of small numbers of idempotents, Michigan Math. J. 14 (1967), 453-465. MR0218922 (36:2006)

[13] B. Prunaru, Fixed points for Lüders operations and commutators, J. Phys. A: Math. Theor. 44 (2011), no. 18, 185203. MR2788723

[14] H. Radjavi and P. Rosenthal, Invariant subspaces (second edition), Dover Publications, Mineola, 2003. MR2003221 (2004e:47010)

[15] V. S. Shulman and Yu. V. Turovskii, Topological radicals, II. Applications to spectral theory of multiplication operators, Elementary Operators and Appl. (R. E. Curto and M. Mathieu, editors), 45-114, Operator Th. Adv. Appl. 212, Birkhäuser, Basel, 2011. MR2789133

[16] J. G. Stampfli, Sums of projections, Duke Math. J. 31 (1964), 455-461. MR0165376 (29:2660)

[17] D. V. Voiculescu, Some results on norm-ideal perturbations of Hilbert space operators, J. Operator Theory 2 (1979), 3-37. MR.553861 (80m:47012) 
[18] L. Weihua and W. Junde, Fixed points of commutative Lüders operations, J. Phys. A 43 (2010), no. 39, 395206. MR2720064 (2011i:81098)

[19] P. Y. Wu, Additive combinations of special operators, Functional Anal. and Operator Theory, Banach Center Publ., vol. 30, Warsaw, 1994, 337-361. MR1285620 (95d:47018)

Department of Mathematics, University of Ljubljana, Jadranska 21, Luubljana 1000, Slovenia

E-mail address: Bojan.Magajna@fmf.uni-lj.si 